\title{
Using linked routinely collected health data to describe prostate cancer treatment in New South Wales, Australia: a validation study
}

\author{
David E Goldsbury ${ }^{1 *}$, David P Smith, Bruce K Armstrong ${ }^{2}$ and Dianne L O'Connell ${ }^{1,2,3,4}$
}

\begin{abstract}
Background: Population-based patterns of care studies are important for monitoring cancer care but conducting them is expensive and resource-intensive. Linkage of routinely collected administrative health data may provide an efficient alternative. Our aim was to determine the accuracy of linked routinely collected administrative data for monitoring prostate cancer care in New South Wales (NSW), Australia.

Methods: The NSW Prostate Cancer Care and Outcomes Study (PCOS), a population-based survey of patterns of care for men aged less than 70 years diagnosed with prostate cancer in NSW, was linked to the NSW Cancer Registry, electronic hospital discharge records and Medicare and Pharmaceutical claims data from Medicare Australia. The main outcome measures were treatment with radical prostatectomy, any radiotherapy, external beam radiotherapy, brachytherapy or androgen deprivation therapy, and cancer staging. PCOS data were considered to represent the true treatment status. The sensitivity and specificity of the administrative data were estimated and relevant patient characteristics were compared using chi-squared tests.

Results: The validation data set comprised 1857 PCOS patients with treatment information linked to Cancer Registry records. Hospital and Medicare claims data combined described treatment more accurately than either one alone. The combined data accurately recorded radical prostatectomy (96\% sensitivity) and brachytherapy (93\% sensitivity), but not androgen deprivation therapy ( $76 \%$ sensitivity). External beam radiotherapy was rarely captured ( $5 \%$ sensitivity), but this was improved by including Medicare claims for radiation field setting or dosimetry (86\% sensitivity). False positive rates were near $0 \%$. Disease stage comparisons were limited by one-third of cases having unknown stage in the Cancer Registry. Administrative data recorded treatment more accurately for cases in urban areas.

Conclusions: Cancer Registry and hospital inpatient data accurately captured radical prostatectomy and brachytherapy treatment, but not external beam radiotherapy or disease stage. Medicare claims data substantially improved the accuracy with which all major treatments were recorded. These administrative data combined are valid for population-based studies of some aspects of prostate cancer care.
\end{abstract}

\section{Background}

Prostate cancer is the most common cancer in New South Wales (NSW), Australia's most populous state, accounting for $19 \%$ of new cancers diagnosed in 2008 [1]. Most men with prostate cancer have localised disease at diagnosis but there is considerable uncertainty regarding their optimal treatment [2]. Population-based

\footnotetext{
* Correspondence: davidg@nswcc.org.au

${ }^{1}$ Cancer Epidemiology Research Unit, Cancer Council NSW, PO Box 572, Kings Cross, NSW 1340, Australia

Full list of author information is available at the end of the article
}

patterns of care studies and disease-specific registers are important for monitoring cancer care but are expensive, resource-intensive and difficult to justify on a continuous basis. Where cancer registries do not collect treatment data, linkage of routinely collected administrative health data to registry records may be an efficient way of monitoring population-wide patterns of cancer care [e.g. [3,4]]. However there is little published information regarding the accuracy of cancer treatment information obtained in this way.

\section{Biomed Central}


The population-based NSW Prostate Cancer Care and Outcomes Study (PCOS) collected data from treating physicians about men who had a first diagnosis of prostate cancer. These data were considered to represent the true treatment status, the "gold standard" of information. This was compared with linked administrative data including cancer registry records, hospital discharge records and Medicare and Pharmaceutical claims data from Medicare Australia. Here we report on the accuracy of the linked, routinely collected administrative health data for describing patterns of prostate cancer care in NSW.

\section{Methods}

\section{Administrative health data}

Data for men who had a first diagnosis of prostate cancer were obtained from the NSW Central Cancer Registry (CCR) [5]. All cancers diagnosed in NSW, except for nonmelanoma skin cancers, are notified to the CCR. Information collected includes date of diagnosis, cancer site, morphology and spread of disease at diagnosis obtained from statutory notification forms and pathology reports. The CCR does not collect treatment data. For this study CCR records were obtained for NSW men diagnosed with prostate cancer between January 1999 and December 2002. Spread of disease was classified as localised, regional spread, distant metastases or unknown according to the system described by Jensen et al [6]. Cases diagnosed after death were excluded from this study.

The NSW Admitted Patient Data Collection (APDC) contains information on all separations from hospital in NSW [7]. Hospital medical coders abstract individual patient information from medical records following the patient's discharge from hospital. This includes dates of admission and separation, procedures carried out and diagnoses relating to the hospital episode. Procedures were coded using the Medicare Benefits ScheduleExtended (MBS-E) classification of the International Classification of Diseases 10th revision, Australian Modification (ICD-10-AM). Diagnosis information was recorded as the reason for the hospital episode, or new or coexisting conditions [8]. Up to 31 procedure codes and 40 diagnosis codes could be recorded for each case. We used hospital separation records from July 1998 to June 2003.

Medicare data [9] included prostate cancer-related claims for medical services from the Medicare Benefits Scheme (MBS) and prescription medicines from the Pharmaceutical Benefits Scheme (PBS), for the period January 2000 to December 2008. These schemes, both components of Australia's national health insurance arrangements, provide subsidised access to medical services and pharmaceuticals for Australian residents.

\section{Patterns of care study data}

PCOS collected data on the patterns of care for 1995 men aged less than 70 years and diagnosed with prostate cancer in NSW between October 2000 and October 2002 [10]. Potential participants were identified through notifications to the CCR. Men who were too ill to be interviewed or who did not speak English were excluded. Ninety-four percent of participants $(n=1883)$ consented to have their information linked to administrative health data. This represents half of the prostate cancers diagnosed in men aged less than 70 years during that period.

Clinical data were abstracted from medical notes at least one year after diagnosis, by either a trained field officer or the treating doctor using a data abstraction form designed for the purpose. Clinical disease stage, radical prostatectomy $(\mathrm{RP})$ and other surgical procedures, radiotherapy and androgen deprivation therapy (ADT) were recorded. Clinical disease stage in PCOS was determined from the clinical data available at diagnosis and was based on biopsy results, prostate-specific antigen levels and digital rectal examination. It was recorded as tumour size, nodal involvement and presence or absence of metastases; these were combined to define localised (tumour size T0-T2c and no known nodal involvement or metastases), non-localised (T3a-T4 or nodal involvement or presence of metastases) or unknown stage.

\section{Record linkage}

The NSW Department of Health, Cancer Institute NSW and Cancer Council NSW ethics committees approved the project and linkage processes. The NSW Department of Health linked the CCR and APDC records using AutoStan version 4.0J to standardise address information and AutoMatch version 4.1 (both Matchware Technologies, Burtonsville, MD, US) to carry out probabilistic matching. This linkage was performed using name, address, date of birth, date of diagnosis and hospital-recorded clinical information that identified cases common to both data sets, and clerical reviews for questionable matches were undertaken by trained staff within the Department of Health. The identifiers from these records were then linked to those from PCOS by the Centre for Health Record Linkage (CHeReL) [11] using probabilistic matching carried out with ChoiceMaker software (ChoiceMaker Technologies Inc., New York, US). Both certain and uncertain matches with PCOS were reviewed clerically by $\mathrm{CHeReL}$ linkage officers, resulting in approximately $0.1 \%$ false positive and less than $0.1 \%$ false negative linkages.

PCOS participants provided their Medicare numbers, allowing for deterministic linkage to MBS and PBS claims data by Medicare Australia (see Figure 1). Participants also provided their name and date of birth on a consent form 


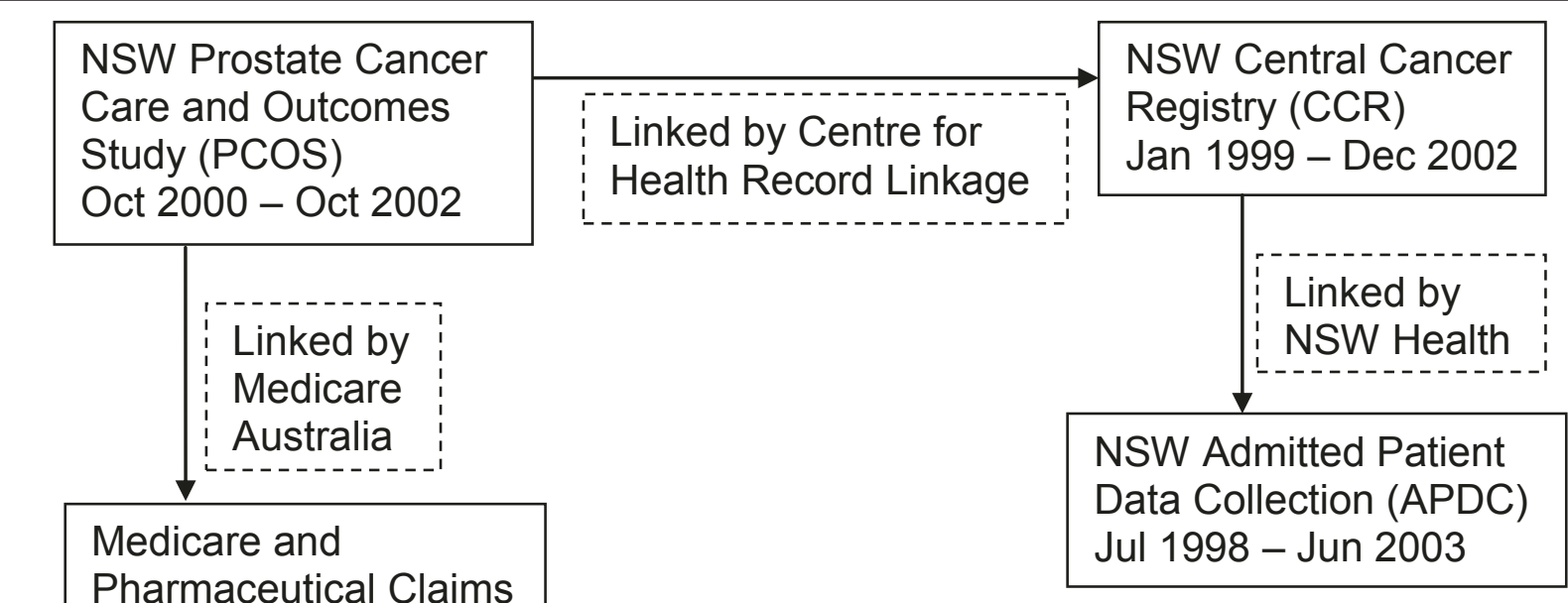

that they signed and dated. If any of these components were missing they were not considered for linkage by Medicare Australia. Furthermore, if the details on the consent form did not match those on the Medicare enrolment database (e.g. the supplied name did not match the name corresponding to the Medicare number on the database) they were not considered for linkage. Additional probabilistic linkage to the MBS and PBS data was not possible.

\section{Treatments and procedures}

A specialist clinical panel identified relevant codes for each data set. Radiotherapy treatment was categorised as external beam radiation therapy (EBRT), brachytherapy, or either one of these ("any radiotherapy"). EBRT and brachytherapy treatment were not mutually exclusive. Diagnosis information from the APDC was also used to identify cases who had radiotherapy based on diagnosis codes for the procedure having taken place (e.g. "Radiotherapy session") or those describing convalescence or sequelae of the treatment (e.g. "Radiation proctitis"). These diagnoses were included as "any radiotherapy", but could not be directly identified as either EBRT or brachytherapy. Other Medicare items related to EBRT (radiation oncologist consultation, pelvic girdle examination, radiation field setting, radiation dosimetry) were included and the change in the accuracy of the administrative data was assessed.

For consistency in the time periods covered by the different data sources we included treatment data up to June 2003. Dates for the receipt of ADT were not recorded in PCOS so we compared all PCOS records to those in the administrative data with and without restriction to June 2003.

\section{Statistical analysis}

Individual patient data from PCOS were compared to the APDC and MBS claims data for RP and radiotherapy procedures, to the PBS claims data for ADT (not captured in the APDC) and to the CCR for disease staging. The sensitivity and specificity of the administrative data relative to PCOS data were estimated. Sensitivity was defined as the probability of an event being recorded in the administrative data if it was recorded by PCOS, while specificity was the probability of an event not being recorded in the administrative data if not recorded by PCOS. Chi-squared tests compared the sensitivity across patient categories defined by age, stage, date of diagnosis, place of residence and socioeconomic status for the postcode of residence, along with selfreported household income, health insurance status and level of education. All analyses were carried out in SAS version 9.1 (SAS Institute Inc., Cary, NC, US).

\section{Results}

Ninety-nine percent $(n=1857)$ of the PCOS participants who consented to linkage to the administrative data were matched to CCR records (Table 1 ). Seventynine percent of these cases linked to records in both the APDC and Medicare claims data, 14\% linked to Medicare claims records only, 6\% linked to APDC records only and $1 \%$ did not link to records in either.

\section{Radical prostatectomy}

The APDC alone captured RPs more accurately than the Medicare data, but combining the two sources improved the accuracy of the administrative data (Table 2). The APDC captured $90 \%$ or more of the RPs for almost all 
Table 1 Characteristics of prostate cancer cases included in this study, as recorded in PCOS $(n=1857)$

\begin{tabular}{lrr}
\hline & $\mathbf{n}$ & \% \\
\hline Age & & \\
$<50$ & 49 & 3 \\
$50-59$ & 627 & 34 \\
$60-69$ & 1181 & 64
\end{tabular}

\begin{tabular}{lrr} 
Disease stage & & \\
Localised & 1617 & 87 \\
Non-localised & 227 & 12 \\
Unknown & 13 & 1 \\
& & \\
Geographical area & & \\
Major city & 1135 & 61 \\
Inner regional & 460 & 25 \\
Outer regional & 235 & 13 \\
Remote/very remote & 22 & 1 \\
Unknown & 5 & 0 \\
& & \\
Radical prostatectomy & 1036 & 56 \\
Any radiotherapy & 578 & 31 \\
$\quad$ EBRT & 530 & 29 \\
$\quad$ Brachytherapy & 120 & 6 \\
Androgen deprivation therapy & 571 & 31 \\
$\quad$ ADT for non-localised cases & 179 & 79 \\
\hline
\end{tabular}

${ }^{a}$ Based on Accessibility/Remoteness Index for Australia (ARIA+), using distance from the place of residence to major service centres PCOS: Prostate Cancer Care and Outcomes Study; EBRT: External beam radiation therapy; ADT: Androgen deprivation therapy

types of patients, with sensitivity lowest for cases living in outer regional areas (78\% vs $93 \%$ for cases living in major cities, $\mathrm{p}<0.0001)$. Sensitivity of the MBS claims data was highest for men who had no private health insurance $(89 \%$ vs $28 \%$ for those with private health insurance, $\mathrm{p}<0.0001)$ and for those living in major cities $(80 \%$ vs $55 \%$ for those living in outer regional areas, $\mathrm{p}<0.0001)$. There were 260 cases with RP recorded in PCOS but not in the MBS claims data, of whom $73 \%$ had data for other MBS claims.

There were 96 men with RP recorded in PCOS but not in the APDC. Sixteen of these had a non-radical prostatectomy recorded in the APDC corresponding to the date of RP recorded in PCOS. Including these other types of prostatectomy, $52 \%$ of cases had prostatectomy recorded in the APDC ( $92 \%$ sensitivity, 100\% specificity). Of the other 80 men, more than half $(n=45)$ did not link to any records in the APDC and 6 cases had a hospital admission at the time of the RP in PCOS but with a different procedure recorded. Forty of the missed cases were treated by clinicians practising outside NSW or in areas bordering on other Australian states. Crossing state borders to receive treatment is common in Australia and is recorded in the hospital data collection of the state providing the treatment. If a patient had a record of care provided by a doctor in an interstate or border region they were flagged as potentially having treatment interstate. After excluding these cases there was no difference in sensitivity of the administrative data by patients' place of residence.

For cases with a RP recorded in both the APDC and PCOS, $85 \%$ had the same date, $13 \%$ were up to a week earlier in the APDC, $1 \%$ were up to a week earlier in PCOS and $1 \%$ had differences larger than this. Similar results were obtained using Medicare data.

\section{Radiotherapy}

While brachytherapy was recorded in the administrative data with reasonable accuracy, the sensitivity with which "any radiotherapy" was recorded was diminished by under-enumeration of EBRT (Table 2). For one quarter of the men with "any radiotherapy" recorded in the APDC, information was obtained from the diagnosis fields only.

Including MBS items for radiation field setting and/or radiation dosimetry (not recorded in the APDC)

Table 2 Accuracy of administrative data sources for ascertaining type of prostate cancer treatment in NSW 2000-2002 ( $n=1857$ )

\begin{tabular}{|c|c|c|c|c|c|c|c|c|c|c|c|c|c|c|}
\hline \multirow[b]{2}{*}{ Procedure } & \multicolumn{2}{|c|}{ PCOS } & \multicolumn{4}{|c|}{ APDC } & \multicolumn{4}{|c|}{ Medicare } & \multicolumn{4}{|c|}{ Medicare + APDC } \\
\hline & $\mathrm{n}$ & $\%$ & $\mathrm{n}$ & $\%$ & Sensitivity & Specificity & $\mathrm{n}$ & $\%$ & Sensitivity & Specificity & $\mathrm{n}$ & $\%$ & Sensitivity & Specificity \\
\hline Radical prostatectomy & 1036 & 56 & 942 & 51 & 91 & 100 & 777 & 42 & 75 & 100 & 996 & 54 & 96 & 100 \\
\hline Any radiotherapy & 578 & 31 & 152 & 8 & 25 & 100 & 114 & 6 & 19 & 100 & 175 & 9 & 29 & 99 \\
\hline Brachytherapy & 120 & 6 & 102 & 5 & 84 & 100 & 99 & 5 & 82 & 100 & 113 & 6 & 93 & 100 \\
\hline EBRT & 530 & 29 & 14 & 1 & 2 & 100 & 21 & 1 & 3 & 100 & 35 & 2 & 5 & 99 \\
\hline EBRT incl. extra items* & 530 & 29 & 14 & 1 & 2 & 100 & 508 & 27 & 86 & 96 & 515 & 28 & 86 & 96 \\
\hline Any RDT incl. extra items* & 578 & 31 & 152 & 8 & 25 & 100 & 523 & 28 & 85 & 98 & 548 & 30 & 89 & 97 \\
\hline Androgen deprivation therapy & 571 & 31 & & & Not recor & ded & 502 & 27 & 76 & 95 & & & & \\
\hline Non-localised cases & 179 & 79 & & & Not recor & rded & 151 & 67 & 82 & 90 & & & & \\
\hline
\end{tabular}

* Extra items are non-treatment visits/procedures comprising radiation field setting and radiation dosimetry

PCOS: Prostate Cancer Care and Outcomes Study; APDC: Admitted Patient Data Collection; EBRT: External beam radiation therapy; RDT: radiotherapy 
dramatically increased the sensitivity with which EBRT was captured with only a small reduction in specificity (Table 2). The improvement was not as great using MBS items for pelvic girdle examination (sensitivity increased to $80 \%, 89 \%$ specificity) or a radiation oncologist consultation ( $85 \%$ sensitivity, $79 \%$ specificity). The sensitivity with which EBRT was recorded was higher for men from major cities $(89 \%)$ compared with those from inner regional areas $(81 \%, \mathrm{p}=0.01)$.

Nineteen cases had brachytherapy recorded in PCOS but did not have curative brachytherapy recorded in the APDC. Of these, 6 had another brachytherapy code in the APDC (single plane brachytherapy or brachytherapy planning), 6 had a "radiotherapy session" recorded in the APDC diagnosis fields on a similar date, 3 had a nonradiotherapy admission recorded on the same date and the remaining 4 cases were treated by doctors practising interstate. Including the other brachytherapy codes increased the sensitivity of the APDC to $89 \%$ and the sensitivity of the combined information from the MBS and APDC to $95 \%$, with no reduction in specificity.

\section{Androgen deprivation therapy}

ADT is more likely to be prescribed for non-localised disease, and the sensitivity of recording in the PBS was higher for this group than for all cases (Table 2). When all PBS items relating to ADT (without date restrictions) were included, the sensitivity increased to $82 \%$ and specificity dropped to $89 \%$ for all cases and was $87 \%$ and $77 \%$ respectively for men with non-localised disease.

\section{Disease stage}

Disease stage was unknown in PCOS for $1 \%$ of men compared to $34 \%$ in the CCR. Localised disease was more likely to be recorded accurately in the CCR than nonlocalised disease (59\% and 19\% sensitivity respectively) (Table 3). Sensitivity of recording of localised disease was highest for younger cases (<50 years: 76\%, 60-69 years: $55 \%, \mathrm{p}=0.0002)$. Of the 1219 cases with known disease stage recorded in both PCOS and the CCR, 90\% were

Table 3 Comparison of disease stage information for cases aged less than 70 years at diagnosis $(n=1844)$

\begin{tabular}{lrrrr}
\hline & & \multicolumn{3}{c}{ PCOS stage $^{\text {a }}$} \\
CCR stage $^{\mathbf{b}}$ & $\mathbf{n}$ & $\begin{array}{r}\text { Localised } \\
\%^{\mathbf{c}}\end{array}$ & $\mathbf{n}$ & \% $^{\mathbf{c}}$ \\
\hline Localised & 958 & 59 & 74 & 33 \\
Non-localised & 143 & 9 & 44 & 19 \\
Unknown & 516 & 32 & 109 & 48 \\
Total & 1617 & 100 & 227 & 100 \\
\hline
\end{tabular}

a Clinical stage from PCOS, excludes 13 cases with unknown stage

${ }^{\mathrm{b}}$ Spread of disease at diagnosis recorded in the CCR

c Column percent

PCOS: Prostate Cancer Care and Outcomes Study; CCR: Central Cancer Registry classified as localised in PCOS compared to $85 \%$ in the CCR (87\% sensitivity, 37\% specificity).

\section{Discussion}

The linked administrative data sets were accurate in enumerating certain prostate cancer treatments. Around 95\% of surgery and brachytherapy treatments, $86 \%$ of EBRT and $76 \%$ of ADT were captured by linking hospital inpatient episodes and Medicare claims data. Supplementing hospital records with Medicare and Pharmaceutical claims data substantially improved the accuracy with which surgery, EBRT, brachytherapy and ADT were captured. Treatment information was more accurate for men resident in urban areas, possibly due to interstate treatment data not being available for men in areas near the state borders.

We assessed the validity of the routinely collected data by comparing them to patients' original medical records. Similar validation studies, including a 1998 NSW breast cancer study [12] and various North American studies [13-16], have also shown administrative data record major surgical procedures well but under-enumerate radiotherapy treatments. Other US studies compared cancer registry and Medicare data for cancer treatment and found high concordance for the records found in both data repositories, but each data repository included patients who were not captured in the other [17-19].

The under-enumeration of radiotherapy treatment is expected as it is generally delivered to outpatients. While Medicare claims accurately identified cases having EBRT, the items used (radiation field setting and dosimetry) generally applied to the initial planning of radiotherapy treatment rather than actual receipt of treatment. While some people identified this way may not have gone ahead with the treatment, the specificity of the administrative data remained over $95 \%$ when these items were included, suggesting that almost all patients did receive treatment. Future studies would benefit from access to data from radiation oncology units or clinical cancer registries.

Disease stage was not recorded in the CCR for one in three prostate cancer cases, significantly limiting interpretation of the appropriateness of prostate cancer care based on disease stage. For cases with known stage recorded in both data sets the agreement was only moderate but it was similar to that reported for colorectal cancer when aggregated to localised or non-localised stage [20].

Non-linkage is a key reason for differences in recorded treatments and there are several possible reasons why PCOS participants were not linked to the administrative data sets. These include not having any inpatient hospital episodes in the APDC (e.g. treated outside NSW, never admitted to hospital), insufficient identifiers to be certain of linkage or the cancer not being registered in the CCR 
within the study period after an early notification record was used in PCOS. An investigation of the 26 men in PCOS who did not have a matching CCR record found a revised diagnosis date may have been outside the study period for 5 cases. PCOS participants provided their Medicare numbers for extraction of their Medicare and Pharmaceutical claims data, but no data were provided for $7 \%$ of these men. The possible reasons for this include incomplete consent forms precluding linkage, supplying an incorrect Medicare number for linkage, or the existence of multiple Medicare numbers for an individual with claims being recorded against a number that was not provided to PCOS.

There are numerous advantages of using administrative data such as these to undertake health services research and to measure the performance of medical and health services. Linkage between data sets can add further value to these resources. Their population coverage ensures large and representative samples are available for study. The data are collected and released on a regular basis, allowing relatively up-to-date monitoring. The costs involved, both in time and resources needed to undertake research, also provide a significant incentive to use linked data rather than intensive data collection from patients or clinicians.

There are some limitations in using these administrative data alone. For example, important factors such as patient performance status, physician recommendations and quality of life are not recorded. Also active surveillance was not explicitly recorded. The data used in this study are now around 10 years old and other treatments are evolving, such as cryotherapy and microwave therapy, and the ability of administrative data to capture this information is not yet known and may be limited. Further validation will be needed as practice changes. More generally, the administrative data were not collected specifically to describe patterns of care so they should be used with caution for this purpose.

Ethical issues may arise regarding linkage of individual's health information. However, projects utilising the Western Australian Data Linkage System [21] have reported several patterns of cancer care studies using similar data sources [e.g. [3,4,22,23]] without raising ethical or privacy-related concerns [24]. It has demonstrated that establishing a privacy-protecting data linkage facility has actually reduced requests for nameidentified information from health data custodians [25].

\section{Conclusions}

Linkage of administrative health data collections to describe the patterns and outcomes of cancer care offers considerable efficiencies in time and resources. The data provided an accurate account of major surgical procedures for prostate cancer in NSW, but were less accurate for
ADT and disease stage and hospital admissions data alone were not sufficient to capture treatment with external beam radiotherapy. Treatment enumeration was substantially improved when hospital records were combined with Medicare and Pharmaceutical claims data. We believe that these administrative data are sufficiently accurate for describing and monitoring surgery and brachytherapy for men with prostate cancer.

\section{Acknowledgements}

We acknowledge the contributions of members of the New South Wales Prostate Cancer Care and Outcomes Study advisory group. We thank the New South Wales Central Cancer Registry for their assistance in the recruitment of study subjects and for making data available for analysis, the NSW Department of Health for providing data and carrying out record linkage, the NSW Centre for Health Record Linkage for conducting record linkage, the National Health and Medical Research Council and the Department of Veterans' Affairs for funding the Prostate Cancer Care and Outcomes Study, the Cancer Institute NSW for partially funding the analysis and the University of Sydney for partially funding the record linkage.

\section{Author details}

${ }^{1}$ Cancer Epidemiology Research Unit, Cancer Council NSW, PO Box 572, Kings Cross, NSW 1340, Australia. ${ }^{2}$ Sydney Medical School, The University of Sydney, NSW 2006, Australia. ${ }^{3}$ School of Public Health and Community Medicine, Faculty of Medicine, University of New South Wales, NSW 2052, Australia. ${ }^{4}$ School of Medicine and Public Health, Faculty of Health, University of Newcastle, NSW 2308, Australia.

\section{Authors' contributions}

DG performed the statistical analysis and drafted the manuscript. DS and BKA guided the analysis and helped to draft the manuscript. DLO designed the study, guided the analysis and helped to draft the manuscript. All authors read and approved the final manuscript.

\section{Competing interests}

The authors declare that they have no competing interests.

Received: 20 December 2010 Accepted: 6 October 2011

Published: 6 October 2011

\section{References}

1. Tracey E, Kerr T, Dobrovic A: Cancer in NSW: Incidence and Mortality Report 2008 Sydney: Cancer Institute NSW; 2010.

2. Wilt TJ, MacDonald R, Rutks I, Shamliyan TA, Taylor BC, Kane RL: Systematic review: comparative effectiveness and harms of treatments for clinically localized prostate cancer. Ann Intern Med 2008, 148(6):435-448, Epub 2008 Feb 4.

3. Hall SE, Holman CDJ, Wisniewski ZS, Semmens J: Prostate cancer: Socioeconomic, geographical and private-health insurance effects on care and survival. BJU Int 2005, 95(1):51-58.

4. Hall SE, Holman CDJ: Inequalities in breast cancer reconstructive surgery according to social and locational status in Western Australia. Eur J Surg Oncol 2003, 29(6):519-525.

5. New South Wales Central Cancer Registry. [http://www.cancerinstitute.org. au/data-and-statistics/cancer-registries/nsw-central-cancer-registry], cited 2011 July 21.

6. Jensen OM, Parkin DM, MacLennan R, Muir CS, Skeet RG: Cancer Registration: Principles and Methods. IARC. Scientific Publications no. 95. Lyon: International Agency for Research on Cancer; 1991.

7. Admitted Patient Data Collection. [http://www.abs.gov.au/AUSSTATS/abs@. nsf/Lookup/1368.1Explanatory\%20Notes1452007?opendocument\&tabname= Notes\&prodno=1368.1\&issue=2007\&num=\&view=], cited 2011 July 21 .

8. National Health Data Committee: Admitted patient care National Minimum Data Set. National Health Data Dictionary, Version 12 Canberra: Australian Institute of Health and Welfare; 2003. 
9. Medicare Australia. [http://www.medicareaustralia.gov.au/about/index.jsp], cited 2011 July 21.

10. Smith DP, King MT, Egger S, Berry MP, Stricker PD, Cozzi P, Ward J, $\mathrm{O}^{\prime}$ Connell DL, Armstrong BK: Quality of life three years after diagnosis of localised prostate cancer: population based cohort study. BMJ 2009, 339: b4817.

11. CHeReL: Centre for Health Record Linkage. [http://www.cherel.org.au], cited 2011 July 21

12. McGeechan K, Kricker A, Armstrong B, Stubbs J: Evaluation of linked cancer registry and hospital records of breast cancer. Aust $N Z J$ Public Health 1998, 22(7):765-770.

13. Malin JL, Kahn KL, Adams J, Kwan L, Laouri M, Ganz PA: Validity of cancer registry data for measuring the quality of breast cancer care. $J$ Natl Cancer Inst 2002, 94(11):835-844.

14. Bickell NA, Chassin MR: Determining the quality of breast cancer care: Do tumour registries measure up? Ann Intern Med 2000, 132(9):705-710.

15. Quan H, Parsons GA, Ghali WA: Validity of procedure codes in International Classification of Diseases, 9th revision, Clinical Modification administrative data. Med Care 2004, 42(8):801-809.

16. Pinfold SP, Goel V, Sawka C: Quality of hospital discharge and physician data for type of breast cancer surgery. Med Care 2000, 38(1):99-107.

17. Cooper GS, Yuan Z, Stange KC, Dennis LK, Amini SB, Rimm AA: Agreement of Medicare claims and tumor registry data for assessment of cancerrelated treatment. Med Care 2000, 38(4):411-421.

18. Cooper GS, Virnig B, Klabunde CN, Schussler N, Freeman J, Warren JL: Use of SEER-Medicare data for measuring cancer surgery. Med Care 2002, 40(Suppl 8):IV43-IV48.

19. Virnig BA, Warren JL, Cooper GS, Klabunde CN, Schussler N, Freeman J: Studying radiation therapy using SEER-Medicare-linked data. Med Care 2002, 40(Suppl 8):IV49-IV54.

20. Yu XQ, O'Connell DL, Gibberd RW, Abrahamowicz M, Armstrong BK: Misclassification of colorectal cancer stage and area variation in survival. Int J Cancer 2008, 122(2):398-402.

21. Holman CDJ, Bass AJ, Rouse IL, Hobbs MST: Population-based linkage of health records in Western Australia: Development of a health services research linked database. Aust N Z J Public Health 1999, 23(5):453-459.

22. Laurvick CL, Semmens JB, Leung YC, Holman CDJ: Ovarian cancer in Western Australia (1982-1998): Trends in surgical intervention and relative survival. Gynecol Oncol 2003, 88(2):141-148.

23. Semmens J, Platell C, Threlfall T, Holman CDJ: A population-based study of the incidence, mortality and outcomes in patients following surgery for colorectal cancer in Western Australia. ANZ J Surg 2000, 70(1):11-18.

24. Giles G: Medical record linkage in Australia: This is as good as it gets. ANZ J Surg 2005, 75(5):259, [Commentary].

25. Trutwein G, Holman CDJ, Rosman DL: Health data linkage conserves privacy in a research-rich environment. Ann Epidemiol 2006, 16(4):279-280.

Pre-publication history

The pre-publication history for this paper can be accessed here:

http://www.biomedcentral.com/1472-6963/11/253/prepub

doi:10.1186/1472-6963-11-253

Cite this article as: Goldsbury et al:: Using linked routinely collected

health data to describe prostate cancer treatment in New South Wales, Australia: a validation study. BMC Health Services Research 2011 11:253.

\section{Submit your next manuscript to BioMed Central and take full advantage of:}

- Convenient online submission

- Thorough peer review

- No space constraints or color figure charges

- Immediate publication on acceptance

- Inclusion in PubMed, CAS, Scopus and Google Scholar

- Research which is freely available for redistribution

Submit your manuscript at www.biomedcentral.com/submit 